\title{
A Study to Ascertain the Effectiveness of a Local DBT Program, Using a Pre-Post Design Model, for Clients in South Kerry Catchment Area of Kerry Mental Health Services (KMHS)
}

Johnson $\mathbf{N}^{*}$

Kerry Mental Health Services, University Hospital Kerry, Tralee, Co. Kerry, Ireland

\begin{abstract}
Aim: The aim of this study was to ascertain whether the local Dialectical Behavior Therapy (DBT) program in South Kerry CMHT was effective in reducing the symptom burden of Borderline Personality Disorder (BPD) along with improving the clients' overall mental health.

Methodology: All the clients who had been participated in the local DBT program were invited in the study. The clients were provided with a detailed account of the rationale, aims and methodology of the study in writing.

The clients were invited to complete 4 self-reported questionnaires: Short-version of Borderline symptom list (BSL-23); Borderline evaluation of severity over time (BEST); Depression Anxiety Stress Scales (DASS) and Satisfaction with Life Scale (SWLS).

The clients were encouraged to pick one week at random, before and after their year-long DBT program, when completing the questionnaires but it was emphasized that it should be the same week for all 4 questionnaires.

Results: There were significant differences between the pre- and post-DBT intervention scores among all scores for the studied patients, which strongly suggested that DBT was an effective intervention for this group.

Conclusions: The results of this study were strongly suggestive that DBT is an effective intervention for clients with BPD. DBT helped the patients to improve on the BPD symptoms but also improved their depressive/anxiety symptoms and their overall satisfaction with life.
\end{abstract}

Keywords: Dialectical behavior therapy (DBT); Borderline personality disorder (BPD); DBT effectiveness; DBT pre-post study design

\section{Introduction}

Dialectical Behavior Therapy (DBT) is a cognitive-behavioral treatment devised by Marsha Linehan to cater to the client group with diagnosis of Borderline Personality Disorder (BPD) [1]. DBT came into being following extensive research into the available literature on psychosocial treatments for other disorders, such as anxiety disorders and depression while attempting to assemble a package of evidencebased, cognitive-behavioral interventions that directly targeted suicidal and self-harm behavior. Most notably, DBT is designed to convey acceptance of the patient and to help the patient accept themselves, their emotions, thoughts, and the world around them, while encouraging and supporting them to change their behaviors and cognitions. This is the classical "dialect" in DBT [2]. Over the years DBT has established itself as one of the fore runners in evidence-based treatments for BPD. Its efficacy has been shown in outpatient settings [3] as well as inpatient settings [4]. The long-term efficacy of DBT has also been established well $[5,6]$. As the evidence grows, professionals have embarked in other areas of use for DBT, with successful results, such as substance use $[7,8]$, eating disorders [9-11] and depression, with a co-morbid personality disorder [12] or without [13]

DBT is a comprehensive program of treatment consisting of skills group training, individual therapy, and a therapist consultation team [14]. In this way, DBT is a program rather than a single treatment method conducted by a practitioner in isolation although there are variations in clinical practice with different models of skills only training or DBT based individual therapy depending on the resources available to clinicians. In all cases, however, it is critical that any adaptation of DBT attempts to fulfil the essential five elements:
1. Improving positive skills, generally through weekly skills group teaching sessions.

2. Generalising skills, using weekly 1:1 sessions with individual therapist.

3. Reducing negative behaviors with a strict hierarchy of tackling life endangering behaviors first, then therapy interfering behaviors and then quality of life interfering behaviors. This can be achieved in 1:1 sessions and through telephone consultations.

4. Supporting the therapist by use of consult groups to avoid therapist burn-out.

5. Structuring the environment so that the skills accrued are not lost by client when they communicate with their environment. This can be achieved by training the clients' families, hospital and A\&E staff in DBT principles.

*Corresponding author: Johnson N, Consultant Psychiatrist, Kerry Mental Health Services, University Hospital Kerry, Tralee, Co. Kerry, Ireland, Tel: 00353877910627; E-mail: navroop.johnson@hse.ie

Received July 13, 2018; Accepted July 20, 2018; Published July 25, 2018

Citation: Johnson N (2018) A Study to Ascertain the Effectiveness of a Local DBT Program, Using a Pre-Post Design Model, for Clients in South Kerry Catchmen Area of Kerry Mental Health Services (KMHS). J Ment Disord Treat 4: 164. doi:10.4172/2471-271X.1000164

Copyright: (c 2018 Johnson N. This is an open-access article distributed under the terms of the Creative Commons Attribution License, which permits unrestricted use, distribution, and reproduction in any medium, provided the original author and source are credited. 


\section{Setting Up of the South Kerry DBT program}

The current DBT program in South Kerry has completed two years and is in session for third year. It was set up after several discussions within the South Kerry team about the ever-present need for this treatment to cater for the clients suffering from BPD and keeping in mind the service burdens these clients were exerting secondary to their difficulties. After various educational presentations to the South Kerry CMHT about the concept of BPD and DBT by the author who is the clinical lead, there was a definite interest expressed by various staff in the team to get trained. This was discussed further with the Management Team for Kerry Mental Health Services (KMHS) and after few discussions, they agreed to support the team in seeking training for DBT.

Training was sought from the initiative of Cork Mental Health DBT team (Endeavour Program) to provide training by bringing DBT Isles (UK) trainers to Cork. This training was funded by the National Office of Suicide Prevention (NOSP). Staff from South Kerry KMHS went to Cork for two separate weeks of training in DBT. After the initial week of Foundation training, the South Kerry DBT program went live in May 2015.

The program was set up on the international format of DBT with a separate Skills Group once a week and patients receiving 1-1 individual therapy along with telephone coaching with their key workers.

\section{Aim}

The aim of this study was to ascertain whether the local DBT program in South Kerry CMHT was effective in reducing the symptom burden of BPD along with improving the clients' overall mental health.

\section{Methodology}

All the clients who had been referred to the local DBT program and had successfully completed it were invited to participate in the study in summer of 2017. The initial contact was made through face to face sessions in OPD or through telephone, in cases where clients had been discharged from the services. The clients were provided with a detailed account of the rationale, aims and methodology of the study. This was also provided to them in writing for future reference. They were assured of anonymization and pooling of the collected data for analysis purposes. They were asked to think about their participation in the study and reply over the next few days. They were encouraged to seek advice and support from their family if they felt like it.

The clients were invited to complete 4 self-reported questionnaires: Short-version of Borderline symptom list (BSL-23) [15]; Borderline evaluation of severity over time (BEST) [16]; Depression Anxiety Stress Scales (DASS) [17] and Satisfaction with Life Scale (SWLS) [18].

The clients were asked to complete each questionnaire considering 1-week duration, before and after completion of their DBT program. It was emphasized to them that each questionnaire was to be filled for a week's symptom duration, and not any longer. They were encouraged to pick one week at random when completing the questionnaires, but it was emphasized that it should be the same week for all 4 questionnaires (Table 1).

The clients were provided with reminders to complete the questionnaires and once all the completed questionnaires were received, they were analyzed using SPSS. The hard copies of the questionnaires were stored in the author's office under lock and key. The electronic

\begin{tabular}{|l|c|c|c|}
\hline \multicolumn{1}{|c|}{ Variable } & Pre-DBT & Post-DBT & p-value \\
\hline \multicolumn{1}{|c|}{$\mathrm{n}=16$} & Median (IQR) & Median (IQR) & \\
\hline BSL-23 & $52.5(41.0-65.5)$ & $11.5(7.3-18.0)$ & $0.001^{\mathrm{w}}$ \\
\hline BSL Overall Personal State (\%) & $30.0(20.0-37.5)$ & $80.0(62.5-80.0)$ & $0.001^{\mathrm{w}}$ \\
\hline BSL Behaviour & $7.0(3.3-20.5)$ & $1.0(0.0-5.5)$ & $0.002^{\mathrm{w}}$ \\
\hline DASS & $40.0(36.3-49.0)$ & $10.5(9.0-15.5)$ & $0.001^{\mathrm{w}}$ \\
\hline SWLS & $8.5(6.3-12.8)$ & $22.0(19.5-27.0)$ & $0.001^{\mathrm{w}}$ \\
\hline BEST Thoughts and Feelings & $30.0(27.0-34.0)$ & $11.0(10.0-13.0)$ & $<0.001^{\mathrm{w}}$ \\
\hline BEST Negative Behaviours & $11.5(8.3-16.8)$ & $4.5(4.0-6.8)$ & $0.001^{\mathrm{w}}$ \\
\hline BEST Positive Behaviours & $6.0(4.3-8.0)$ & $13.0(11.3-14.7)$ & $0.002^{\mathrm{w}}$ \\
\hline wWilcoxon Signed Ranks Test & & & \\
\hline
\end{tabular}

Table 1: Comparison of self-reported questionnaires, pre and post DBT intervention

data was stored on a HSE encrypted laptop, using password protected files (word, excel and SPSS).

\section{Description of Questionnaires Used}

BSL-23 is a shorter version of the BSL-95 which was developed by Prof. Martin Bohus of Germany. The BSL-95 was developed as a selfrating instrument to specifically quantify BPD symptomatology. The items are based on the criteria of the DSM-IV, the opinions of clinical experts and borderline patients. The psychometric properties and validity of the BSL have been investigated in several studies.

The Borderline Evaluation of Severity Over Time (BEST) was developed to rate the thoughts, emotions, and behaviors typical of BPD, in the University of Iowa by Bruce Pfohl and Nancee Blum. This is a Likert-like scale that includes 15 items and three subscales. The first eight items comprise subscale A (Thoughts and Feelings), and involve assessments of mood reactivity, identity disturbance, unstable relationships, paranoia, emptiness, and suicidal thinking. The next four items comprise subscale B (Behaviors-Negative), which rates negative actions such as injuring oneself. Items on these subscales are rated from 1 (None/Slight) to 5 (Extreme). The final three items comprise subscale $\mathrm{C}$ (Behaviors-Positive), which rates actions such as following through on therapy plans. These items are rated from 5 (Almost Always) to 1 (Almost Never). The instrument showed moderate test-retest reliability, high internal consistency, and high discriminant validity.

The DASS is a set of three self-report scales designed to measure the negative emotional states of depression, anxiety and stress. Each of the three DASS scales contains 14 items, divided into subscales of 2-5 items with similar content. The Depression scale assesses dysphoria, hopelessness, devaluation of life, self-deprecation, lack of interest/ involvement, anhedonia, and inertia. The Anxiety scale assesses autonomic arousal, skeletal muscle effects, situational anxiety, and subjective experience of anxious affect. The Stress scale is sensitive to levels of chronic non-specific arousal. It assesses difficulty relaxing, nervous arousal, and being easily upset/agitated, irritable/over-reactive and impatient. Subjects are asked to use 4-point severity/frequency scales to rate the extent to which they have experienced each state over the past week. Scores for Depression, Anxiety and Stress are calculated by summing the scores for the relevant items.

The SWLS is a short 5-item instrument designed to measure global cognitive judgments of satisfaction with one's life.

\section{Results}

A total of 23 clients had been in receipt of the DBT program in South Kerry between 2015 and 2017 (two sessions). Out of these, 2 clients were discharged from the services as they had relocated to other counties. Telephone contact was unsuccessful with both. 5 clients 
refused to participate in the study. Out of the 5 who refused, 3 had not completed the program and were displaying ongoing cognitive and behavioural dysregulation which perhaps would explain their reticence for participation. This left 16 clients who participated in the study. Out of the 16 clients, 13 were females and 3 males. The age range was between 19 and 72, with the mean age as 38.4 years. As for their relationship status, $34 \%$ of the clients were married, $24 \%$ were single and $42 \%$ were separated.

Initially the raw data was cleaned and scores from the BEST, SWLS, DASS, and BSL questionnaires were input into one flat data file and imported into SPSS v24. Individual question scores were given for the BEST questionnaire while cumulative scores were given for the other three questionnaires. Because the BEST questionnaire is divided into three parts (Thoughts \& Feelings, Negative Behaviours, and Positive Behaviours), cumulative scores were calculated for each of these categories for both pre- and post-DBT intervention.

Nonparametric methods were required in this analysis due to the small sample size $(n=16)$ and skewed nature of the scores. The Wilcoxon Signed Ranks test was used to compare scores pre- and postintervention.

There were significant differences between the pre- and postDBT intervention scores among all scores for the studied patients. The improvement was not only noted in the symptoms specific to BPD, which were demonstrated by the score difference in BSL-23 and BEST but also in DASS scores which account for depression, anxiety and stress levels. The clients in the study also felt that they were more satisfied with their lives.

\section{Discussion}

This study is an attempt to evaluate the effectiveness of a local DBT program within Kerry Mental Health Services, based in the south of Ireland. In line with research evidence for DBT, the author felt that the local program also succeeded in reducing the negative symptom burden for the clients whilst improving their positive behaviours and overall satisfaction with life. As hypothesised by the author, the degree of change in the rating scales for the BPD symptoms was significant and was supported by the statistical analysis results. As far as the author is aware, there isn't another study in Ireland looking at real life effectiveness of DBT using a pre-post study design.

A study by Dr. Janet Feigenbaum of University College London (UCL), published in British Journal of Clinical Psychology in 2012 demonstrated symptomatic improvement in a cohort of 42 patients, where the therapy was delivered by NHS staff that didn't have extensive training [19]. Our study would be similar in the level of training for the DBT therapists. Feigenbaum, et al. study compared DBT with TAU (Treatment as usual) and employed the RCT methodology, whereas this study was based on a pre-post study design with no comparison with TAU. Other differences were the assessments at start, 6 months and 1 year of intervention for their study versus our study where both the pre and post questionnaires were completed following the intervention. The results from our study are much more encouraging though but the number of participants was smaller [19].

Like the above research in UCL, Christian Stiglmayr and colleagues published the first-year results from the Berlin Borderline study in 2014, which attempts to show effectiveness and generalizability for DBT in an outpatient setting [20]. This study started with 78 patients but 47 completed the first year of therapy. The attrition rate is higher for them than it was for our study. For the patients who completed the therapy though, there was a good (77\%) rate of improvement in their psychopathology. This would be a similar finding to our study. The colleagues from Berlin have looked at service related outcomes as well like inpatient bed numbers which we didn't. Our study did look at co-morbid symptom burden though and showed a very positive improvement in this area. The power and design of the two studies are not comparable though.

Our study also showed a statistically significant improvement in depressive and anxiety symptoms which would mirror the findings of the study published in the American Journal of Geriatric Psychiatry by Lynch and colleagues [13], although that study involved a different age group of clients and the comparison was between a group which received antidepressants alone versus a group that received DBT with antidepressants. The results in the latter group were statistically better and stayed better at 6 months follow up as well.

Overall the author believes that even though this study involves a relatively smaller cohort of patients from one service in south of Ireland, the results add to the credence for the effectiveness of DBT. The study also adds to the growing evidence that DBT has use in improvement of affective symptoms and is a therapy that can provide a group of dysregulated patients improved coping behaviors and satisfaction with life.

\section{Limitations}

Even though this study demonstrated what it set out to achieve, the author feels that there are some biases with the study, which can be improved upon in future works. A self-selected sample of patients from one psychiatric practice limits the interpretation of results and may create a bias.

With patients retrospectively assessing their behaviors and quality of life to answer the pre-DBT questionnaires, memory bias may be introduced as patients may exaggerate their scores pre-DBT in comparison to how they feel post-DBT. Ideally a pre-intervention questionnaire about the patients' previous week would be given before DBT is undertaken, then a post-intervention questionnaire about the patients' previous week would be given at least one week after DBT is undertaken. As the author of the study was also the treating physician for the participants, a possibility of social desirability bias cannot be ignored.

\section{Conflict of Interest statement}

Navroop Johnson has no conflicts of interest to disclose.

\section{Ethical Standards Statement}

The author asserts that all procedures contributing to this work comply with the ethical standards of the relevant national and institutional committee on human experimentation with the Helsinki Declaration of 1975, as revised in 2008. The author asserts that ethical approval for publication of this survey was not required by their local Ethics Committee.

\section{Financial Supports Statement}

This research received no grant from any funding agency, commercial or notfor-profit sectors.

\section{References}

1. Linehan MM, Armstrong HE, Suarez A, Allmon D, Heard HL (1991) Cognitivebehavioral treatment of chronically parasuicidal borderline patients. Arch Gen Psychiatry 48: 1060-1064.

2. Linehan MM, Tutek DA, Heard HL, Armstrong HE (1994) Interpersonal outcome of cognitive behavioral treatment for chronically suicidal borderline patients. Am J Psychiatry 151: 1771-1776.

3. Lynch TR, Chapman AL, Rosenthal MZ, Kuo JR, Linehan MM (2006) 
Citation: Johnson N (2018) A Study to Ascertain the Effectiveness of a Local DBT Program, Using a Pre-Post Design Model, for Clients in South Kerry Catchment Area of Kerry Mental Health Services (KMHS). J Ment Disord Treat 4: 164. doi:10.4172/2471-271X.1000164

Page 4 of 4

Mechanisms of change in dialectical behavior therapy: theoretical and empirical observations. J Clin Psychol 62: 459-480.

4. Kroger C, Schweiger U, Sipos V, Arnold R, Kahl KG, et al. (2006) Effectiveness of dialectical behavior therapy for borderline personality disorder in an inpatient setting. Behav Res Ther 44: 1211-1217.

5. Van den Bosch LMC, Koeter MW, Stijnen T, Verheul R, Van den Brink W (2005) Sustained efficacy of dialectical behavior therapy for borderline personality disorder. Behav Res Ther 43: 1231-1241.

6. Linehan MM, Comtois KA, Murray AM, Brown MZ, Gallop RJ, et al. (2006) Twoyear randomized controlled trial and follow-up of dialectical behavior therapy vs therapy by experts for suicidal behaviors and borderline personality disorder. Arch Gen Psychiatry 63: 757-766.

7. Linehan MM, Dimeff LA, Reynolds SK, Comtois KA, Welch SS, et al. (2002) Dialectical behavior therapy versus comprehensive validation therapy plus 12-step for the treatment of opioid dependent women meeting criteria for borderline personality disorder. Drug Alcohol Depend 67: 13-26.

8. Soler J, Pascual JC, Campins J, Barrachina J, Puigdemont D, et al. (2005) Double-blind, placebo-controlled study of dialectical behavior therapy plus Olanzapine for borderline personality disorder. Am J Psychiatry 162: 12211224.

9. Safer DL, Telech CF, Agras WS (2001) Dialectical behavior therapy for bulimia nervosa. Am J Psychiatry 158: 632-634.

10. Safer DL, Lock J, Couturier JL (2007) Dialectical behavior therapy modified for adolescent binge eating disorder: A case report. Cogn Behav Pract 14: 157167.

11. Salbach-Andrae H, Bohnekamp I, Pfeiffer E, Lehmkuhl U, Miller AL (2008) Dialectical behavior therapy of anorexia and bulimia nervosa among adolescents: a case series. Cogn Behav Pract 15: 415-425.
12. Lynch TR, Cheavens JS, Cukrowicz KC, Thorp SR, Bronner L, et al. (2007) Treatment of older adults with co-morbid personality disorder and depression: a dialectical behavioral therapy approach. Int J Geriatr Psychiatry 22: 131-143.

13. Lynch TR, Morse J, Mendelson T, Robins C (2003) Dialectical behavior therapy for depressed older adults: a randomized pilot study. Am J Geriatr Psychiatry 11: 33-45.

14. Linehan MM (1993) Cognitive-Behavioral Treatment of Borderline Personality Disorder. Guilford Press, New York

15. Bohus M, Kleindienst N, Limberger MF, Stieglitz RD, Domsalla M, et al. (2009) The Short Version of the Borderline Symptom List (BSL-23): Development and Initial Data on Psychometric Properties. Psychopathology 42: 32-39.

16. Pfohl B, Blum N, St. John D, McCormick B, Allen J, et al. (2009) Reliability and validity of the borderline evaluation of severity over time (BEST): A self-rated scale to measure severity and change in persons with borderline personality disorder. J Pers Disord 23: 281-293.

17. Lovibond SH, Lovibond PF (1995) Manual for the Depression Anxiety Stress Scales. Psychology Foundation, Sydney.

18. Diener E, Emmons RA, Larsen RJ, Griffin S (1985) The Satisfaction with Life Scale. J Pers Assess 49: 71-75.

19. Feigenbaum JD, Fonagy $P$, Pilling $S$, Jones A, Wildgoose A, et al. (2012) A real-world study of the effectiveness of DBT in the UK national health service. Br J Clin Psychol 51: 121-141.

20. Stiglmayr C, Stecher-Mohr J, Wagner T, Meibner J, Spretz D, et al. (2014) Effectiveness of dialectic behavioral therapy in routine outpatient care: The Berlin Borderline Study. Borderline Personal Disord Emot Dysregul 1: 20. 